\title{
Antibiotic resistance in patients suffering from nosocomial infections in Besat Hospital
}

\author{
Sirous Faraji Hormozi (1), Narges Vasei (2), Mohammad Aminianfar (3), Mohammad Darvishi \\ (3), Ali Asghar Saeedi (1) \\ (1) Infectious Diseases and Tropical Medicine Research Center (IDTMRC), AJA University of \\ Medical Sciences, Tehran, Iran; (2) Department of Surgery, Besat Hospital, AJA University of \\ Medical Sciences, Tehran, Iran; (3) Infectious Diseases and Tropical Medicine Research \\ Center (IDTMRC), Department of Aerospace and Subaquatic Medicine, AJA University of \\ Medical Sciences, Tehran, Iran
}

This article is distributed under the terms of the Creative Commons Attribution Noncommercial License (CC BY-NC 4.0) which permits any noncommercial use, distribution, and reproduction in any medium, provided the original author(s) and source are credited.

\begin{abstract}
This study was performed to determine the trend of antibiotic resistance of the causative organisms among the patients suffering from nosocomial infections in Besat Hospital since 2013 to 2015. In this observational study that was performed as a retrospective cohort, 935 consecutive patients with nosocomial infection were enrolled in Besat Hospital since 2013 to 2015. The trend of antibiotic resistance of the causative organisms among them was determined and contributing factors were assessed. The finding of this study revealed that type of microorganisms had significant variation $(\mathrm{p}=0.024)$ : while the gram-negative bacilli have shown an increased level of resistance, the gram positive cocci had less resistance. The antibiotic resistance was increased for ampicillin/sulbactam, piperacillin/tazoactam, ceftriaxone, ceftazidime, cefepime, meropenem, gentamicin, amikacin, ciprofloxacine, levofloxacine, nitrofurantoin, and ampicilline. However, it was decreased for colistin. In conclusion, antibiotic resistance has an increasing trend and strategic measures of prevention are needed to reduce nosocomial infections.
\end{abstract}

Key Words: Antibiotic resistance, trend, nosocomial infection, health care

Eur J Transl Myol 28 (3): 304-308, 2018

Hospital infections and antibiotic resistance are problems that have been reported for many years around the world, causing the monetary burden and prolonged treatment. An epidemiological study on the incidence of hospital infections in the United States showed that, the risk of infectious diseases has increased steadily. A total of 2 million people are affected by a hospital infection, where it is being imposed on the health system at a cost of $\$ 4.5$ to $\$ 11$ billion. In addition, 80,000 deaths annually occur due to hospital infections. ${ }^{1}$ Hospital infections are caused by hospitalization 48 hours after the patient admission or 30 days after being discharged from the hospital. Nosocomial infections are one of the most common types of infections. ${ }^{2}$ These infections are mainly caused by bacterial agents, such as Staphylococcus, Pseudomonas and Escherichia coli, and are more often observed as pneumonia and urinary tract infections (UTI), in 22 patients per 1,000 people. ${ }^{2}$ However, the main cause of infection is not detected in $17 \%$ of patients. ${ }^{2}$ It is worth noting that Staphylococcus aureus is the most common form of pneumonia and Escherichia coli in cases of UTI. ${ }^{3}$ Of course, the rates and types of hospital infections are different in various hospitals, where the rates of UTI, pneumonia, and bacteremia are the most common types in some hospitals. $^{4}$ Therefore, their therapeutic pattern is different in treatment centers where is dominated by predominant microorganisms and resistant types. Antibiotic resistance can be initial or acquired. Resistant agents are generally bacteria that have high virulence, such as Staphylococcus aureus. Also, antibiotic resistance is related to their history of use. The most important steps in preventing hospital infections are identifying the factors that affect these infections and taking precautionary measures based on the use of appropriate strategies. ${ }^{5}$ In this regard, In this regard, the establishment of surveillance systems is important to track the trend of hospital infections ${ }^{6}$. The importance and necessity of this is especially more for antibiotic resistance because, according to available reports, this 
Table 1. Frequency distribution of the agent type in studied years

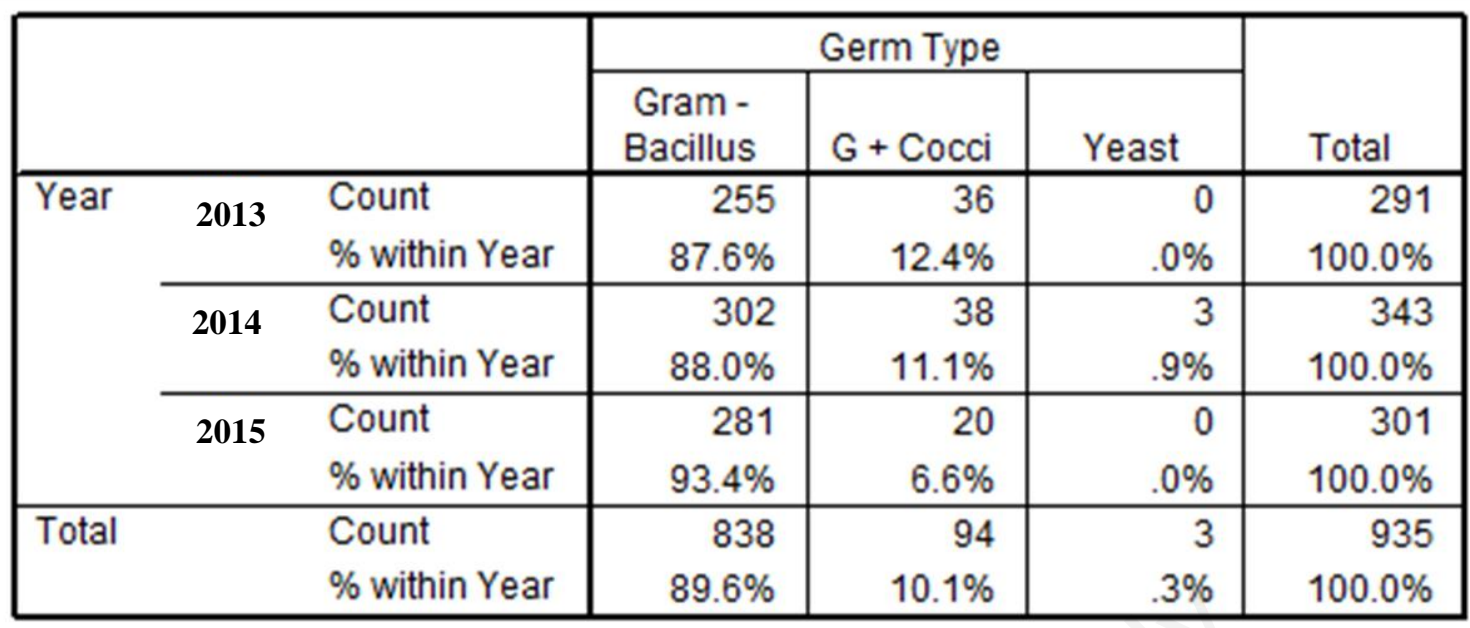

issue is rising. By identifying the pattern of antibiotic resistance changes, it is possible to provide suggestions on how antibiotics are administered, and how to change their course, as well as empirical treatment. Therefore, the present study was aimed to investigate the change in the pattern of antibiotic resistance in the microorganisms causing nosocomial infections during the years 2013 to 2015 in Besat Hospital.

\section{Materials and Methods}

This observational study was conducted as a retrospective cohort study. A total of 935 patients with nosocomial infections during the years 92 to 94 were selected and examined in Besat Hospital. Also, antibiotic resistance patterns were studied in microorganisms based on antibiogram and their relationship with other variables was evaluated. Data were analyzed using SPSS software version 13. Mean and standard deviation were determined for quantitative variables while absolute and relative frequency was recorded for qualitative variables. Chi-Square, Fisher, T-independent and ANOVA test were used to evaluate the variables. A $\mathrm{p}$ value $<0.05$ was considered significant.

\section{Results and Discussion}

In the present study, a total of 935 patients with nosocomial infections were evaluated, from of which 514 cases $(55 \%)$ were male and $421(45 \%)$ were female. Sex distribution was not statistically significant in the studied years $(\mathrm{p}>0.05)$. The mean age of patients in studied years was $63.9 \pm 36.18$ to $66.38 \pm 29.18$ years. The frequency of age distribution of patients did not show a significant difference $(\mathrm{p}>0.05)$. Regarding to the type of cultivation, 631 cases belonged to sputum culture $(67.5 \%)$, followed by 49 blood cultures $(5.2 \%)$, 187 urine culture (20\%), 68 ulcer culture $(7.3 \%)$. The distribution of the type of patient sample did not show a significant difference $(\mathrm{p}>0.05)$. Regarding the frequency of disease type, 363 cases were related to Acinetobacter (38.8\%), followed by E coli (207 cases; $22.1 \%)$, Staphylococcus aeruginosa (140; 15\%), Staphylococcus aureus (50; 5.3\%), and other agents including coagulase-negative Staphylococci, Enterobacter, Klebsiella, and Candida. The type of agent in the studied years was found to be significantly different $(\mathrm{p}=0.001)$, so that pseudomonas decreased and Acinetobacter showed an increased incidence. Regarding the frequency of resistance to vancomycin, 5 cases $(93.7 \%)$ were susceptible to vancomycin and 7 cases $(6.3 \%)$ were resistant. Frequency of vancomycin resistance in patients was not significantly different in studied years $(p>0.05)$. Regarding the frequency of oxacillin resistance, 30 cases $(42.7 \%)$ were susceptible to oxacillin and 40 were resistant. There was no significant difference in the frequency of oxacillin resistance in patients during the studied years $(p>0.05)$. Resistance to ampicilin / sulbactam was seen in 152 cases $(39 \%)$, while 238 cases $(61 \%)$ were susceptible. Distribution of ampicillin / sulbactam resistance in patients in studied years was demonstrated to be significant, where had U pattern $(\mathrm{p}=0.014)$. Moreover, the resistance of piperacillin / tazobactam was seen in 272 cases $(56 \%)$, while $214(44 \%)$ were susceptible. It is worth noting that resistance to piperacillin / tazobactam in patients showed a significant level where it showed an incremental pattern $(\mathrm{p}=0.037)$. Furthermore, 32 cases $(43.2 \%)$ revealed resistance to cefazolin, while $42(56.8 \%)$ were susceptible. The frequency of resistance to cefazolin did not show significant difference among patients in studied years ( $\mathrm{p}$ $>0.05)$. In addition, frequency of cefoxitin resistance was found in 25 cases (43.9\%), however, 32 cases $(56.1 \%)$ were susceptible to this antibiotic. There was no significant difference in frequency distribution of 
Antibiotic resistance of nosocomial infections

Eur J Transl Myol 28 (3): 304-308, 2018

Table 2. Pattern of antibiotic resistance based on age of patients

\begin{tabular}{ll}
\hline Year & Antibiotic resistance \\
\hline 2013 & Age was not associated with any antibiotic resistance $(\mathrm{p}>0.05)$. \\
2014 & Senior age had a significant correlation with Clindamycin resistance $(\mathrm{p}=0.044)$. \\
2015 & The higher age group had a significant association with resistance to gentamicin $(\mathrm{p}=0.018)$ \\
& and the age was significantly correlated with clindamycin resistance $(\mathrm{p}=0.034)$. \\
& \\
& Table 3. Change in the pattern of antibiotic resistance based on gender of patients \\
\hline Year & Antibiotic resistance \\
2013 & Female gender was associated with antimicrobial resistance to amikacin $(\mathrm{P}=0.049)$. \\
2015 & Male sex was associated with antimicrobial resistance to levofloxacin $(\mathrm{P}=0.012)$. \\
\hline
\end{tabular}

cefoxitin resistance in patients during the studied years ( $\mathrm{p}>0.05$ ). Our findings demonstrated that 63 cases $(11.9 \%)$ were susceptible to ceftriaxone and 466 $(88.1 \%)$ were resistant to ceftriaxone. The frequency of resistance to ceftriaxone showed significant changes, indicating an incremental pattern $(\mathrm{p}=0.010)$. With regard to the frequency of resistance to ceftazidime, 49 cases $(9.9 \%)$ revealed susceptibility to this antibiotic, while $445(90.1 \%)$ confirmed antibiotic resistance. As a result, significant changes were found in the frequency of resistance to ceftazidime in patients, where an incremental pattern was achieved $(\mathrm{p}=0.001)$. Regarding resistance to cefepime, 103 cases (20.2\%) showed sensitivity and $406(79.8 \%)$ were resistant. The frequency of resistance to imipenem among patients was significantly higher in studied years and had an incremental pattern $(\mathrm{p}=0.001)$. In the studied years, 132 cases $(24.8 \%)$ were sensitive to imipenem and 400 cases $(75.2 \%)$ showed resistance. The frequency of imipenem resistance in patients in studied years did not reveal a significant difference $(\mathrm{p}>0.05)$. Moreover, Resistance to meropenem was also found in 353 cases $(67.5 \%)$, but the sensitivity of this biotype was found in $170(32.5 \%)$ patients. The frequency of resistance to meropenem in patients demonstrated a significant change in studied years and had a reverse U pattern ( $\mathrm{p}=$ 0.002 ). With regard to the frequency of resistance to gentamicin, 88 cases $(23.1 \%)$ were found to be susceptible while 293 cases $(66.9 \%)$ were identified to be resistant. The frequency of resistance to gentamicin in patients revealed significant changes over the years and was U-shaped pattern $(\mathrm{p}=0.002)$. In this study, other antibiotics such as amikacin, ciprofloxacin, levofloxacin, nitrofurantoin, clostin, and ampicillin have been evaluated. Frequency of resistance in patients in studied years revealed significant changes where u- shaped patterns were observed regarding amikacin, ciprofloxacin, levofloxacin.It should be noted that in the case of clostine, a decreasing resistance pattern was demonstrated and an incremental resistance pattern was determined for ampicillin. It is worth noting that there were no significant differences in resistance to antibiotics such as clindamycin, rifampicin, clotrimazole, linezolid and nalidixic acid in studied years. On the other hand, diversity of antibiotic resistance patterns based on age, gender type of sample and type of agent in the subjects are listed listed in Tables 2 to 5 . The findings of this study revealed that the frequency distribution of the agent type had significant changes $(\mathrm{P}=0.024)$ : gram negative bacilli increased notably, while gram positive cocci decreased (Table 1). The trends in antibiotic resistance pattern of bacterial agents based on the age of the subjects was significant in few cases $(\mathrm{p}<0.05)$ that are shown in table 2 . The change in antibiotic resistance pattern based on gender was also significant in few cases (Table 3; p $<0.05$ ). The trend of changing the patterns of antibiotic resistance based on the type of sample was significant in some cases $(p<0.05)$, that are shown in Table 4 . In addition, there was a significant relationship between the trend of antibiotic resistance pattern and type of agent in most cases (Table 5; $\mathrm{p}<0.05$ ).

Establishing surveillance systems for tracking the trend of infectious diseases is of particular importance. ${ }^{6}$ This is especially true for antibiotic resistance because it is increasing among patients. By identifying the pattern of antibiotic resistance, suggestions can be made on how antibiotics are administered, so the study was designed to assess the changes in antibiotic resistance pattern among microorganisms involved in nosocomial infections. Microorganisms had significant variation ( $\mathrm{p}$ $=0.024)$. The gram-negative bacilli have shown an 
Table 4. Antibiotic resistance pattern based on sample type

\begin{tabular}{|c|c|}
\hline Year & Antibiotic resistance \\
\hline 2013 & $\begin{array}{l}\text { The blood sample was associated with antibiotic resistance to vancomycin }(\mathrm{p}=0.001) \text {, } \\
\text { oxacillin }(\mathrm{p}=0.021) \text {, ceftriaxone }(\mathrm{p}=0.002) \text {, cefipime }(\mathrm{p}=0.013) \text {, linzolide }(\mathrm{p}=0.003) \text { and } \\
\text { clindamycin }(\mathrm{p}=0.015) \text {. A sample of phlegm was associated with antibiotic resistance to } \\
\text { piperacillin / tazobactam }(\mathrm{p}=0.001) \text {, meropenem }(\mathrm{p}=0.001) \text {, amikacin }(\mathrm{p}=0.001) \text { while } \\
\text { the urine sample was related to antibiotic resistance to nalidixic acid }(\mathrm{p}=0.001) \text {. }\end{array}$ \\
\hline 2014 & $\begin{array}{l}\text { Blood samples were correlated with antibiotic resistance to ceftriaxone }(\mathrm{p}=0.001) \text {, } \\
\text { Cefepime }(\mathrm{p}=0.001) \text {, meropenem }(\mathrm{p}=0.001) \text {, amikacin }(\mathrm{p}=0.001) \text {, and colistin }(\mathrm{p}= \\
0.040) \text {. The association of sputum samples with antibiotic resistance was detected in a } \\
\text { number of bacteria including piperacillin / tazobactam }(\mathrm{p}=0.001) \text {, ceftazidime }(\mathrm{p}=0.043) \text {, } \\
\text { imipenem }(\mathrm{p}=0.001) \text {, gentamicin }(\mathrm{p}=0.001) \text {, and ciprofloxacin }(\mathrm{p}=0.001) \text {. }\end{array}$ \\
\hline 2015 & $\begin{array}{l}\text { The association of blood samples with antibiotic resistance was observed only in ceftriaxone } \\
(\mathrm{p}=0.001) \text { and cefepime }(\mathrm{p}=0.001) \text {. Furthermore, the sputum samples had a significant } \\
\text { relationship with antibiotics in terms of drug resistance including piperacillin / tazobactam } \\
(\mathrm{p}=0.001) \text {, imipenem }(\mathrm{p}=0.001) \text {, moropenem }(\mathrm{p}=0.001) \text { and amikacin }(\mathrm{p}=0.001) \\
\text { ciprofloxacin ( } \mathrm{pp}=0.027) \text { and levofloxacin }(\mathrm{p}=0.016) \text {. While urine specimens were } \\
\text { associated with antibiotic resistance to co-trimoxazole }(\mathrm{p}=0.005)\end{array}$ \\
\hline
\end{tabular}

Table 5. Change in antibiotic resistance patterns based on the type of agents

\begin{tabular}{ll}
\hline Year & Antibiotic resistance \\
\hline 2013 & $\begin{array}{l}\text { There was correlation between agents and antibiotic resistance for all antibiotics }(\mathrm{p}<0.05) \text { other } \\
\text { than vancomycin, linezolid and cotrimoxazole }\end{array}$ \\
& There was a correlation between agents and antibiotic resistance for all antibiotics $(\mathrm{p}<0.05)$. \\
2014 & $\begin{array}{l}\text { A significant association was found between agents and antibiotic resistance in all antibiotics (p }< \\
\text { 0.05) with the exception of oxacillin, cefazolin, cefoxitin, nalidixic acid, nitrofurantoin, rifampicin } \\
\text { and clindamycin }\end{array}$ \\
\hline
\end{tabular}

increased level of resistance, while the gram positive cocci had less resistance. The antibiotic resistance was increased for ampicillin / sulbactam, piperacillin / tazoactam, ceftriaxone, ceftazidime, cefepime, meropenem, gentamicin, amikacin, ciprofloxacine, levofloxacine, nitrofurantoin, and ampicilline. However, it was decreased for colistin. Behzadnia et al. 2014, ${ }^{7}$ evaluated the nosocomial infections in children in north of Iran for identifying the antibiotic sensitivity of their causative organisms. They reported that all the gram positive and negative bacterial isolates revealed remarkable resistance to antibiotics. Moreover, multidrug-resistannce of Acinetobacter spp. was found by the mentioned study. ${ }^{6}$ Pseudomonas spp. (36.84\%) and Acinetobacter spp. (28.02\%) were mostly found in isolated samples. However, pseudomonas showed a significant decrease in our research, while an increase in the frequency of Acinetobacter was reported. Necati Hakyemez et al. in Turkey assessed nosocomial $A$. baumannii antibiotic resistance in patients suffering from nosocomial infections. ${ }^{8}$ The most effective antibiotics in isolated strains included imipenem, meropenem, colistin and tigecycline, as reported previously by Necati Hakyemez et al. ${ }^{8}$ However, they showed that the antibiotic resistance level against imipenem and meropenem has increased over the years, an observation that our research findings confirm. Another study demonstrated antibiotic-resistant Acinetobacter Baumannii infections in another Hospital in Tehran, Iran. As reported by Vahdani et al, ${ }^{9}$ the highest resistance belonged to ceftazidime (96\%), followed by ceftizoxime (95\%), ceftriaxone (93\%), ciprofloxacin (85\%), co-terimoxazole $(85 \%)$, gentamicin $(68 \%)$, amikacin $(58 \%)$ and imipenem $(9 \%)$. Report that gram-positive bacteria, in particular Staphylococcus aureus, have a 100-percent resistance to ceftriaxone, cotrimoxazole and cefotaxime, while they have a high sensitivity to vancomycin. There was also a high resistance among gram-negative bacteria to the antibiotics investigated in mentioned study, including ceftriaxone, cefotaxime and cotrimoxazole. ${ }^{9}$ Accordingly, the frequency of agents involved in nosocomial infections will vary from region to region. ${ }^{4,9,10}$. In our study, the type of microorganism influence antibiotic resistance. Weinstein et al. ${ }^{11}$ in the United States indicated that antibiotic resistance of the causative organisms is increasing among the patients with nosocomial infections, specificlly for staphylococci, and enterobacteriaceae (Pseudomonas aeruginosa), as we confirm with present findings. We 


\section{Antibiotic resistance of nosocomial infections}

Eur J Transl Myol 28 (3): 304-308, 2018

may concluded that antibiotic resistance is increasing in nosocomial infections in our Hospital. New strategic measures are needed to reduce them by prevention programs. It is recommended that further studies are performed to confirm our results by higher sample size and multicenter approch. Furthermore, investigations are needed on the factors that affect antibiotic resistance.

\section{List of acronyms}

UTI - Urinary tract infection

\section{Author's contributions}

Authors contributed equally to the manuscript.

\section{Acknowledgments \\ Funding: None.}

\section{Conflict of Interest}

The authors declare no conflicts of interests.

\section{Ethical Publication Statement}

We confirm that we have read the Journal's position on issues involved in ethical publication and affirm that this report is consistent with those guidelines.

\section{Corresponding Author}

Ali Asghar Saeedi, Infectious Diseases and Tropical Medicine Research Center (IDTMRC), Aja University of Medical Sciences, Tehran, Iran. Phone +98 9032721145

Email: dr.saeedi@gmail.com

\section{E-mail of co-authors}

Sirous Faraji Hormozi: Syrous.faraji@yahoo.com NargesVasei:nvasei@yahoo.com Mohammad Aminianfar: maminianfar@yahoo.com Mohammad Darvishi: Darvishi1349@gmail.com

\section{References}

1. Faraji Hormozi S, Saeedi AA, Aminianfar M, Salmani Alishah M, Darvishi M. Studying the Frequency of Nosocomial Infection and its Relative Factors in the Intensive Care Unit of Hospitals Based Upon NNI System. Eurasian J Anal Chem 2018;13:em16.

2. Ak O, Batirel A, Ozer S, Çolakoglu S. Nosocomial infections and risk factors in the intensive care unit of a teaching and research hospital: a prospective cohort study. Med Sci Monit 2011;17:29-34.
3. Darvishi M. Antibiotic Resistance Pattern of Uropathogenic Methicillin - resistant Staphylococcus aureus Isolated from Immunosuppressive Patients with Pyelonephritis. J Pure Apple Microbe 2016;10:2663-667.

4. Richards MJ, Edwards JR, Culver DH, Gaynes RP. Nosocomial infections in medical intensive care units in the United States. National Nosocomial Infections Surveillance System. Crit Care Med 1999;27:887-92.

5. Manzoni P, De Luca D, Stronati $M$, et al. Prevention of nosocomial infections in neonatal intensive care units. Am J Perinatol 2013;30:81-8.

6. Emori TG, Gaynes RP. An overview of nosocomial infections, including the role of the microbiology laboratory. Clin Microbiol Rev 1993;6:428-42.

7. Behzadnia S, Davoudi A, Rezai MS, Ahangarkani F. Nosocomial infections in pediatric population and antibiotic resistance of the causative organisms in north of Iran. Iran Red Crescent Med J 2014;16:e14562.

8. Necati Hakyemez I, Kucukbayrak A, Tas T, et al. Nosocomial Acinetobacter baumannii Infections and Changing Antibiotic Resistance. Pak J Med Sci 2013;29:1245-8.

9. Vahdani P, Yaghoubi T, Aminzadeh Z. Hospital acquired antibiotic-resistant acinetobacter baumannii infections in a 400-bed hospital in Tehran, Iran. Int J Prev Med 2011;2:127-30.

10. Ahmed SH, Daef EA, Badary MS, Mahmoud MA, Abd-Elsayed AA. Nosocomial blood stream infection in intensive care units at Assiut University Hospitals (Upper Egypt) with special reference to extended spectrum beta-lactamase producing organisms. BMC Res Notes 2009;2:76.

11. Weinstein RA. Controlling antimicrobial resistance in hospitals: infection control and use of antibiotics. Emerg Infect Dis 2001;7:188-92.

Submission: June 5, 2018

Revision received: June 14, 2018

Acceptance: June 14, 2018 